\title{
Temporal excitation patterns on the cerebral cortex as a result of migraine modeling
}

Julia M. Kroos, Ibai Diez, Jesus M. Cortes, Sebastiano Stramaglia, and Luca

Gerardo-Giorda

\begin{abstract}
The complex, highly individual, geometry of the cerebral cortex in humans presents a major challenge in studying the spreading of spontaneous neuronal activity. Recent computational advances [1] allow to simulate the propagation of depolarization waves on the macroscale and for individual geometries, reconstructed from accurate medical imaging as MRI, with high levels of detail. In this paper we take advantage of such technique to study the temporal excitation patterns that follow the passage of a depolarization wave on the cerebral cortex.
\end{abstract}

\section{Introduction}

One of the most intriguing phenomenon related to reaction-diffusion processes in complex geometries is the spreading depression of spontaneous activity, initially discovered and characterized in animal cortices $[2,3]$. Nowadays these phenom-

Julia M. Kroos

Basque Center for Applied Mathematics, Bilbao, Basque Country, Spain

e-mail: jkroos@bcamath.org

Ibai Diez

Biocruces Health Research Institute, Cruces University Hospital, Barakaldo, Spain

e-mail: ibaidiez85@gmail.com

Jesus M. Cortes

Biocruces Health Research Institute, Barakaldo, Spain and Departamento de Biologia Celular e Histologia, University of the Basque Country, Leioa, Spain and Ikerbasque: The Basque Foundation for Science. Bilbao, Spain

e-mail: jesus.m.cortes@gmail.com

Sebastiano Stramaglia

Dipartimento di Fisica, Università di Bari, Italy, and INFN, Sezione di Bari, Italy

e-mail: sebastiano.stramaglia@ba.infn.it

Luca Gerardo-Giorda

Basque Center for Applied Mathematics, Bilbao, Basque Country, Spain

e-mail: lgerardo@bcamath.org 
ena are believed to be relevant in a plethora of brain pathologies, such as cerebral ischemia-infarction, transient global amnesia, epilepsy or migraine auras, the situation we focus on here. Several experiments suggest that a propagating depolarization wave on the cortex is underlying migraine, see $[4,5,6]$ and references therein. This wave, named cortical spreading depression (CSD), causes a drastic failure of the brain homeostasis and is followed by a wave of inhibition. CSD is characterized by relevant increases in both extracellular $\mathrm{K}+$ and glutamate, as well as rises in intracellular $\mathrm{Na}+$ and $\mathrm{Ca} 2+$. These ionic shifts result in slow direct current potential shifts that can be recorded extracellularly. Starting in the visual cortex, CSD propagates to the peripheral areas. It is worth mentioning that, whilst CSD has been experimentally demonstrated in animal models, the strongest evidences for CSD in humans are the migraine aura itself, and the spreading oligemia phenomenon observed in migraine patients, consisting in a decrease of the cerebral blood flow in the posterior part of the brain at the beginning of the attack, followed by the spreading of the low flow region at a speed which is similar to those found for CSD in animals. Some electrophysiological evidences that human grey matter in vivo supports CSD have also been reported (see, e.g., [7]). A deeper understanding of CSD phenomenology would be useful to test current and novel prophylactic drugs, while providing knowledge on mechanisms of action relevant for migraine [8]. The role played by the peculiar geometry of the brain cortex in supporting or blocking CSD is far from being satisfactorily understood. In a recent paper [1] we investigated several aspects of the brain geometry which favor or disfavor the propagation of CSD. In particular, by using a computational neuronal model distributed throughout a realistic cortical mesh, we have found significant differences in the propagation traveling patterns of CSD, both intra and inter-hemispherically, and revealed important asymmetries in the propagation profile. Moreover we identified brain regions displaying a peculiar behavior during CSD propagation, in particular, some regions that appear to trap the propagating action potentials for a longer time. Those regions play a key role in the CSD propagation (and possibly would be able to stop it). These results are relevant to identify target structures for therapies using stereotactic cortical neuromodulation, see e.g. [9].

In the present work we will concentrate on another feature of the propagating CSD wave, still exploiting the computational frame of simulations on the realistic cortical mesh. In the specific, we will study the temporal patterns of excitation associated with the propagation of the CSD by analyzing the percentage of cortex that can get excited at the same time. The paper is organized as follows. In Section 2 we describe the distributed model for CSD propagation, its numerical approximation and the characteristics of the realistic cortical computational grid. In Section 3 the temporal activity we study in this paper is introduced. Finally, numerical results from the simulation of the distributed model are collected in Section 4. 


\section{Modeling and numerical simulation of CSD on real geometries}

\subsection{A distributed computational model for CSD propagation}

A key property of neural cells is to produce an action potential (AP). It consists in a sudden variation in the transmembrane potential, called spike, followed by a recovering of the resting condition through a refractory period, during which the cell cannot be excited. In agreement with previous computational studies [10], we consider neurons at rest to have a background firing rate of $4 \mathrm{~Hz}$ while excited neurons fire with an average frequency of $64 \mathrm{~Hz}$.

In [1] we introduced a slow variables model for the firing rate to describe the neuronal activity, where the state variable $u(x, t)$ represents the average firing rate of neurons at location $x$ and time $t$ (in seconds). Such model is locally a temporal mean field one with respect to the finer scale of the action potential, and represents the typical all-or-nothing response of a single excited cell in a simplified manner [11]. The model is a modification of the Roger-McCulloch variant of the FitzhughNagumo model [12], that has been adapted to reproduce the characteristic neuronal activity, with a resting state of $4 \mathrm{~Hz}$, a firing rate of $64 \mathrm{~Hz}$ in the excited state, and the plateau length matching the duration of the neuron excitation after the passage of the CSD (around 10 minutes, [13]).

Table 1 Model parameters.

\begin{tabular}{lll}
\hline Parameter & Value & Description \\
\hline$G$ & 0.2667 & \\
$u_{0}$ & 4 & resting value of the firing rate \\
$u_{t h}$ & 11.8 & threshold value for excitation \\
$u_{p}$ & 64 & peak value of the firing rate \\
$\eta_{1}, \eta_{2}, \eta_{3}$ & $0.4806,3.3333 \mathrm{e}-5,60$ & \\
\hline
\end{tabular}

A diffusion term accounts for the spatial propagation of the excitation on the cortex, and the complete model reads:

$$
\begin{aligned}
\frac{\partial u}{\partial t} & =-I(u, w)+\operatorname{div}(D \nabla u) \\
I(u, w) & =G\left(u-u_{0}\right)\left(1-\frac{u}{u_{t h}}\right)\left(1-\frac{u}{u_{p}}\right)+\eta_{1}\left(u-u_{0}\right) w \\
\frac{\partial w}{\partial t} & =\eta_{2}\left(u-u_{0}-\eta_{3} w\right)
\end{aligned}
$$

where $u(x, t)$ is the firing rate at time $t \geq 0$, and $w(x, t)$ is the recovery variable, $u_{t h}$ and $u_{p}$ are threshold and peak values for $u, u_{0}$ is the background firing rate and $D \in \mathbf{R}^{3 \times 3}$ is the conductivity tensor, while $\eta_{1}, \eta_{2}, \eta_{3}$ and $G$ are parameters, whose values are given in Table 1 . The coupled PDE-ODE system (1) is defined 
on the computational domain $\Omega \times(0, T), \Omega \subset \mathbf{R}^{3}$. To ensure that problem (1) is mathematically well posed, initial conditions $u^{0}(x)=u(x, 0), w^{0}(x)=w(x, 0)$ must be imposed in $\Omega$ and boundary conditions on $\partial \Omega$. If the computational domain is a 2D surface $\Sigma \subset \mathbf{R}^{3}$ the classical divergence and gradient operators are replaced by their tangential counterparts $\operatorname{div}_{\Sigma}$ and $\nabla_{\Sigma}$. Boundary conditions are not necessary if the surface $\Sigma$ is closed, as in the case of the reconstructed cortical geometry we consider in the numerical simulations of this paper.

\subsection{Numerical approximation}

We discretise problem (1) by $\mathbf{P}_{1}$ finite elements in space and finite differences in time (for an introduction to finite element methods see, e.g. [14]). Let $t^{n}=n \Delta t$, for $n=0, \ldots, N$, be a discretisation of the time interval $(0, T)$ : we denote with $u^{n}$ and $w^{n}$ the approximation of $u$ and $w$ at time $t^{n}$. We use an implicit-explicit (IMEX) scheme to advance from time $t^{n}$ to $t^{n+1}$. The recovery variable $w^{n+1}$ can be solved explicitly and is used in the expression $I(u, w)$ to compute $u^{n+1}$. The overall scheme reads:

$$
\begin{aligned}
& \text { update: } & w^{n+1} & =\frac{u^{n}-u_{0}}{\eta_{3}}+\left(w^{n}-\frac{u^{n}-u_{0}}{\eta_{3}}\right) \exp \left(-\eta_{2} \eta_{3} \Delta t\right) \\
& \text { update: } & I^{n+1} & =I\left(u^{n}, w^{n+1}\right) \\
& \text { solve: } & A u^{n+1} & =M u^{n}-\Delta t M I^{n+1}
\end{aligned}
$$

where $A:=M+\Delta t S$, while $M$ and $S$ are the classic finite elements mass and stiffness matrices.

\subsection{Computational domain}

A crucial aspect in studying the behaviour of an individual brain relies on the use of personalized computational domains. The cortical geometry we used in this study has been reconstructed from a MRI scan with FreeSurfer image analysis suite (http://surfer.nmr.mgh.harvard.edu/): for further details, see [15] and references therein. This data set was already acquired and published in [16], and used in [1], and its acquisition was approved by the Ethics Committee at the Cruces University Hospital: all the methods employed were in accordance to approved guidelines. The data set corresponds to one healthy subject, male, age 28 , and was acquired with a Philips Achieva 1.5T Nova scanner. The cortical mesh was obtained from a highresolution anatomical MRI, acquired using a T1-weighted 3D sequence with the following parameters: $\mathrm{TR}=7.482 \mathrm{~ms}$, $\mathrm{TE}=3.425 \mathrm{~ms}$; parallel imaging (SENSE) acceleration factor $=1.5$; acquisition matrix size $=256 \times 256$; $F O V=26 \mathrm{~cm}$; slice thickness $=1.1 \mathrm{~mm} ; 170$ contiguous sections. 
The computational grid is a triangulation of the reconstructed cortex, consisting of 140,208 nodes and 280,412 triangles for the left hemisphere and 139,953 nodes and 279,902 triangles for the right hemisphere.

\subsubsection{Regions of interest}

We consider a subdivision of the brain cortex into different regions of interest (ROIs): we base our study on the anatomical subdivision of each hemisphere into 34 ROIs, which is a generalised version of the Brodmann atlas [17] (included in the MRIcro software http://www.mricro.com).

\subsection{Simulation protocol}

The numerical simulations of equations (1) are performed with a self-developed code in Matlab (MathWorks Inc., Natick, MA), taking advantage of its Parallel Computing Toolbox to speed up the computations on multicore processors. We chose a uniform time step of $\Delta t=0.6$ seconds, and an isotropic diffusion tensor $D=\delta$ Id, with $\delta=0.7174 \mathrm{~mm}^{2} \mathrm{~s}^{-1}$. The conductivity coefficient has been tuned to ensure that a wave is actually propagating across the cortex, at a velocity comparable with the one of the CSD.

The finite element matrix $A$ associated with the problem is poorly conditioned, entailing high costs in terms of CPU time. In order to speed up the computation, a proper preconditioning strategy can be implemented, such as an incomplete Cholesky factorisation with threshold, pivoting and properly chosen drop tolerance [18]. Since the linear system is symmetric, a Preconditioned Conjugate Gradient (PCG) is the natural choice as a solver.

CSD is known to originate from the visual cortex, but to gain deeper insight in the effect of geometry on the propagation we simulate, in both hemispheres, the spread of excitation waves between all the regions of the anatomical classification. In each simulation, we consider as initial condition one fully depolarized region out of the 34 in the anatomical subdivision. Each simulation is run until all remaining regions have been fully activated. The only compartment that is not considered as initially activated is the corpus callosum as it constitutes the intersection between the two hemispheres and obeys different rules for diffusion.

\section{Temporal activity}

We are interested in studying the pattern of excited regions over the course of time. Although CSD is originating in the visual cortex, we aim for a deeper understanding of the geometry impact on the propagation when a depolarization wave is starting 

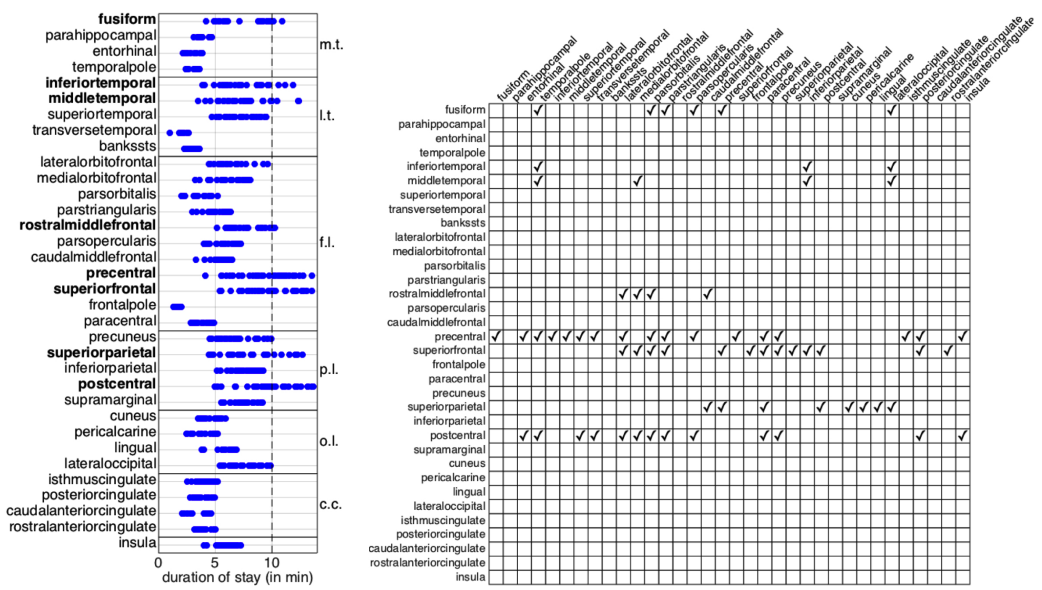

Fig. 1 Duration of stay of the depolarisation wave and regions of the left hemisphere featuring a long residence time. The duration of stay of the excitation wave in the different regions for all the initially excited regions possible (left). The dashed line draws the 10 minutes threshold; regions featuring waves staying longer than 10 minutes are marked in bold. The abbreviations give the affiliation to the lobes: m.t. medialtemporal lobe, 1.t. lateraltemporal lobe, f.l. frontal lobe, p.l. parietal lobe, o.l. occipital lobe, c.c. cingulate cortex. A detailed description of the initially activated regions causing the wave to stay for longer than 10 minutes are marked with a tick (right). Rows represent the arrival regions, columns the initially activated ones.

from another area. In that order, we initialise the excitation in one of the 34 different regions of the brain, and observe the number of regions that is excited at every point in time. The first aspect to consider is the definition of an activated region. Since the duration of the excitation following the passage of CSD is about 10 minutes [13], some caution is mandatory with regions that are either big or feature a large aspect ratio. In such regions, it might occur that some of the points initially excited might have already gone back to resting state before every remaining point in the region gets excited. In view of these remarks, we consider a region as excited at a given moment in time if $80 \%$ of the grid points located in it are excited. Before taking a more detailed look at any excitation pattern we thus have to verify that every region we are considering can be deemed excited at a given moment in time, independently from the initially activated area.

As done in [1], we record, for all simulations, the minimum and the maximum activation times (in minutes) for each of the 33 regions that were not excited at $t=0$. The minimum activation time is the moment when the first point of the region at hand gets excited, while the maximum activation time is the moment at which the last point of the region gets excited. Such values are collected into four $34 \times 34$ matrices, that we denote by $L_{\min }, L_{\max }, R_{\min }$ and $R_{\max }$, where $L$ and $R$ refer to the left and right hemisphere, respectively. To emphasize the spatial connection between the regions, the ordering of the regions is arranged according to their affiliation to 


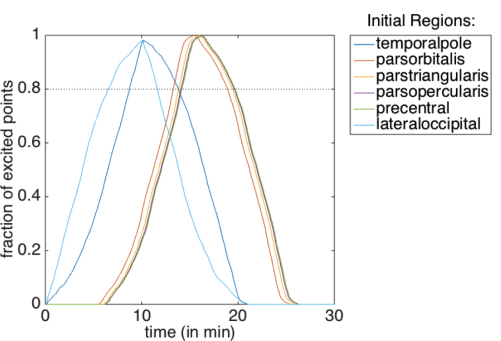

(a) fusiform

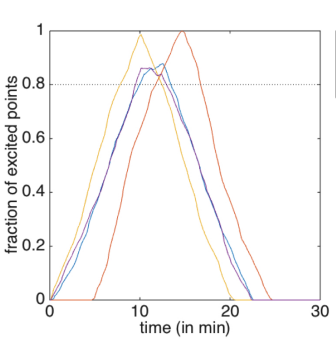

(c) middletemporal

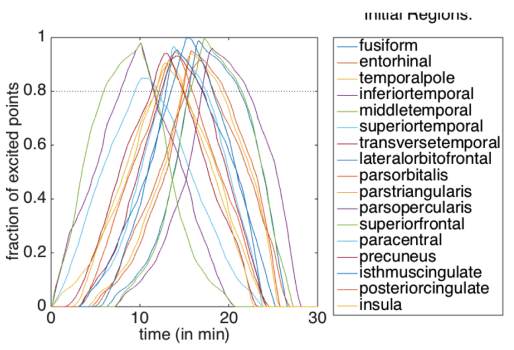

(e) precentral

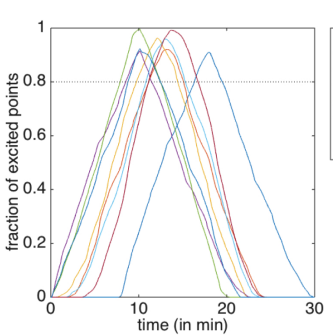

(g) superiorfrontal

Initial Regions: caudalmiddlefronta precentral
paracentral paracentral cuneus lingual ateraloccipital

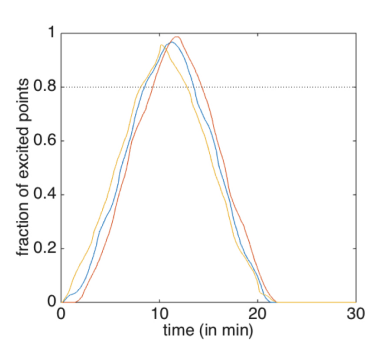

(b) inferiortemporal

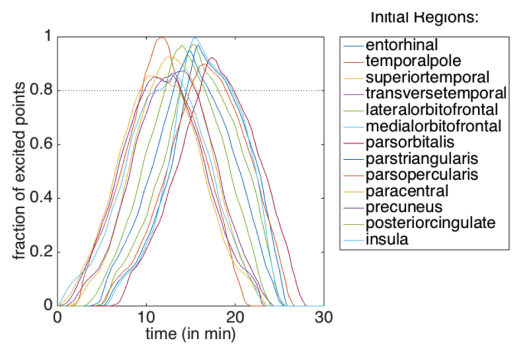

(d) postcentral

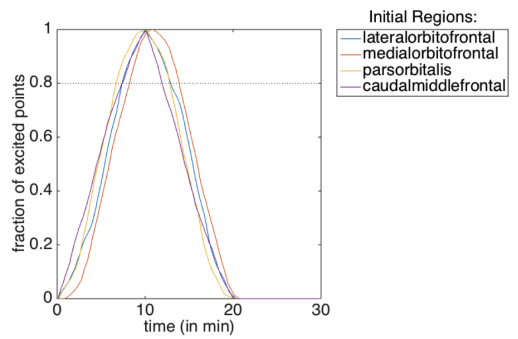

(f) rostalmiddlefrontal

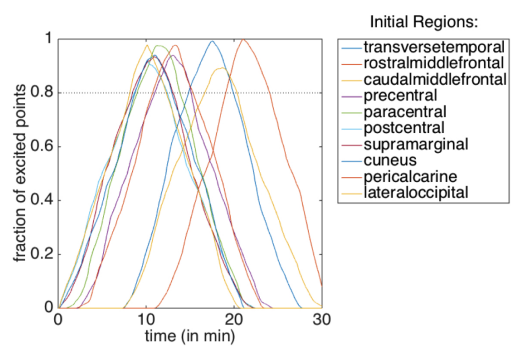

(h) superiorparietal Initial Regions: - inferiorparietetal

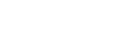

Fig. 2 Detailed excitation patterns for the regions of the left hemisphere featuring a long residence time. The percentage of excited points in time in the regions (a) - (h) identified in Figure 1 for the different initially excited regions. The dotted line marks the threshold value of $80 \%$, at which the whole region is considered excited. 
lobes. Regions belonging to one lobe are then clustered according to the mutual distance of their centroids in the Euclidean norm. In all of the above matrices, rows represent the starting region of the wave propagation, while columns the arrivals: as an example, the $(i, j)$-th element of $L_{\min }$ represents the arrival time in region $j$ of a wave originated in region $i$. We also introduce the depolarization residence matrices by considering the difference between the maximum and minimum activation times (or equivalently, the time elapsed between the depolarization of the first and the last point of the region), as

$$
D^{L}=L_{\max }-L_{\min } \quad D^{R}=R_{\max }-R_{\min } .
$$

Again, rows represent the starting region of the wave propagation, while columns the difference between activation times: the $(i, j)$-th element of $D^{L}$ and $D^{R}$ represents the residence time in region $j$ of a wavefront originating from region $i$. For illustrative purpose, we focus here on the left hemisphere, but results and conclusions similar to the ones we present in the following can be drawn for the right hemisphere as well. Whenever $D_{i, j}^{L}>10$, the region $j$ might not be considered as excited by a wave originating in region $i$. In Figure 1 (left), we plot the columns of the residence matrix $D^{L}$, where the different markers in each row of the plot correspond to different initially excited regions. The threshold of 10 minutes, after which a region might not get excited as a whole at any given time, is highlighted by a dashed line, and the 8 regions featuring residence time longer than 10 minutes are marked in bold. These regions are fusiform, inferiortemporal, middletemporal, rostralmiddlefrontal, precentral, superiorfrontal, superiorparietal, and postcentral. In Figure 1 (right) we pair these 8 peculiar regions with the corresponding initially activated ones: rows indicate the arrival regions, columns the initially activated areas, while checkmarks highlight the occurrencies when the depolarisation wave lasts longer than $10 \mathrm{~min}-$ utes. The regions so identified in Figure 1 require a more detailed analysis to check whether $80 \%$ of their grid points are excited at any point in time. For each of these regions, we computed the percentage of activated points along time for a depolarization originating in the areas identified in Figure 1 (right). A detailed visualisation of these patterns is given in Figure 2, from which we can infer that all the regions feature a period in time where at least $80 \%$ of their points is excited, independently of the origin of the initial excitation. Analogous results hold for the 9 regions of the right hemisphere (the same as the ones in the left hemisphere, plus the precuneus one) featuring a residence time longer than 10 minutes.

Since we ensured that all regions get fully excited independently from the initially activated region, we can focus on the temporal activation patterns. Given an initially activated region, we simulate the propagation of the depolarization wave and monitor along time the percentage of excited points in every arrival region, thus identifying the number of excited region at every moment in time. 


\section{Results}

A comparison for the two hemispheres for all the possible initially excited regions can be performed. Although the two hemispheres globally feature similar excitation schemes in the course of time, some differences can be spotted. In order to exhibit the typology of such differences, we display in Figure 3 the comparison between the two hemispheres for 6 representative regions, one per lobe: fusiform, middletemporal, medialorbitofrontal, postcentral, lingual and rostralanteriorcingulate. The major differences we observe are in the guise of similar excitation patterns that are shifted in time, where one of the two hemispheres features a faster activation than the other, like for the medialorbitalfriontal (c) and the rostralanteriorcingulate (f), or in the form of similar temporal patterns, but pretty different excitation ones, like in the case of fusiform (a), middletemporal (b), postcentral (d), and lingual (e).

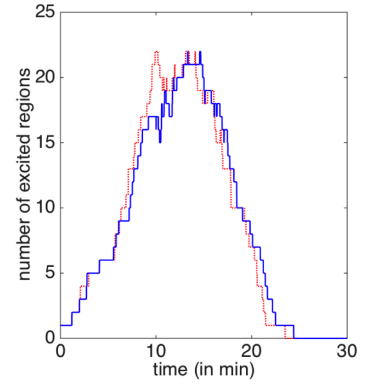

(a) fusiform

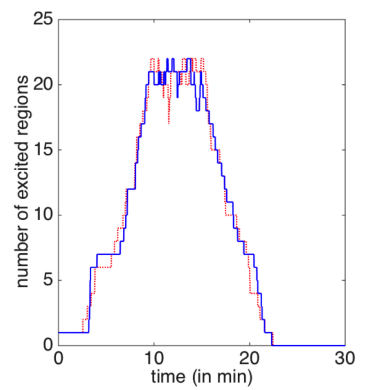

(d) postcentral

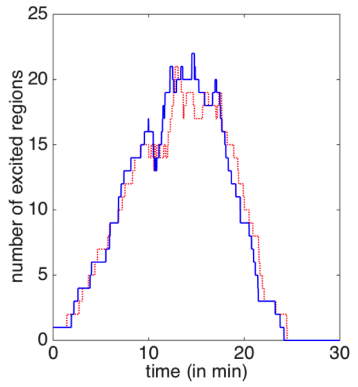

(b) middletemporal

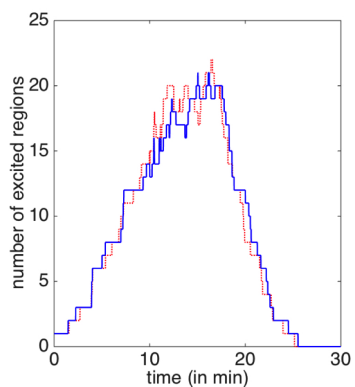

(e) lingual

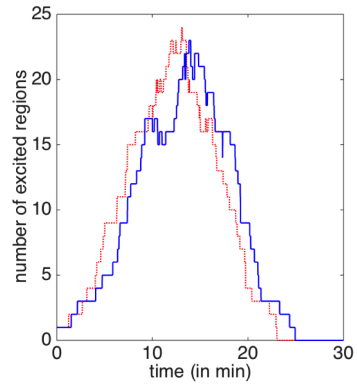

(c) medialorbitofrontal

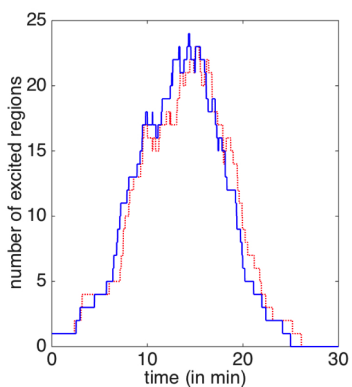

(f) rostalanteriorcingulate

Fig. 3 Number of excited regions. The number of excited regions with initially activated regions (a) - (h) for the left (red) and right (blue) hemisphere; each one is chose exemplarily for each lobe.

For a more global approach, we average the number of excited regions over all the initially excited areas at each time step, obtaining a temporal excitation pattern for the left and right hemisphere. These temporal excitation patterns are shown in Figure 4. We display in the background (in blue) the excitation patterns for every 

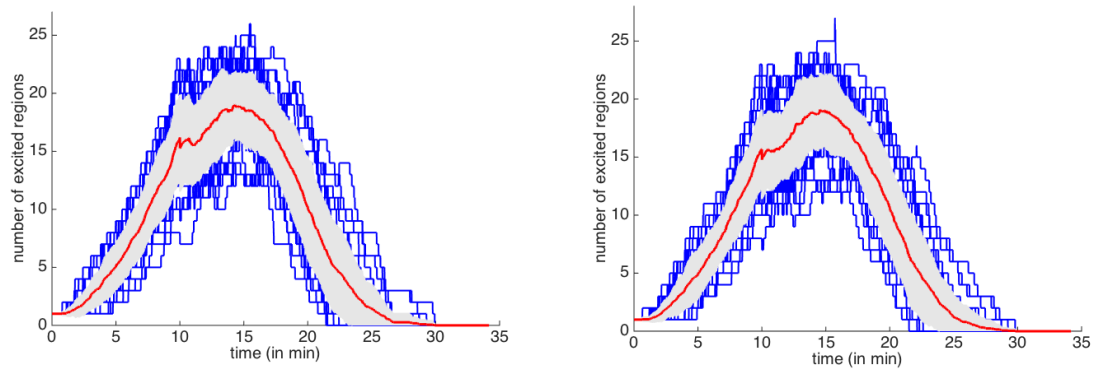

Fig. 4 Average number of excited regions. The average number of excited regions (red) - averaged over all initially activated regions - for the left (a) and right (b) hemisphere. For all initially excited regions the number of excited regions is plotted in blue and the grey area marks the standard deviation around the average.

initial region and we highlight (in grey) the standard deviation around the mean. The temporal excitation patterns of the two hemispheres are globally very similar, but show some differences in the vicinity of 10 minutes after the start of the wave propagation.

The maximum number of excited regions for each initially activated area highlights their impact on the excitation pattern of the cortex. A comparison of the left and right hemisphere is given in Figure 5, where a significant variation in the maximum number of activated regions is more evident intra-hemispherically than interhemispherically. As an example, a depolarization wave originating from the lateraloccipital area will excite at once only up to 16 regions (in both hemispheres), whereas a wave originating from the posteriorcingulate area can activate up to 27 regions in the left hemisphere ( 25 in the right hemisphere). Left and right hemisphere exhibit a difference of up to 2 regions, but 10 areas feature exactly the same maximum number of excited regions in the two hemispheres.

\section{Conclusions}

It has been recently proposed that thalamocortical dysrhythmia may be responsible for the altered synchronicity in migraine; CSD is expected to play a central role in migraine pathogenesis being the cause of the aura phenomenon. In order to gain further insights of the role of geometry in the temporal excitation patterns following the passage of a depolarization wave on the cerebral cortex, here we have adopted a distributed computational model for CSD, and studied the temporal pattern con- 


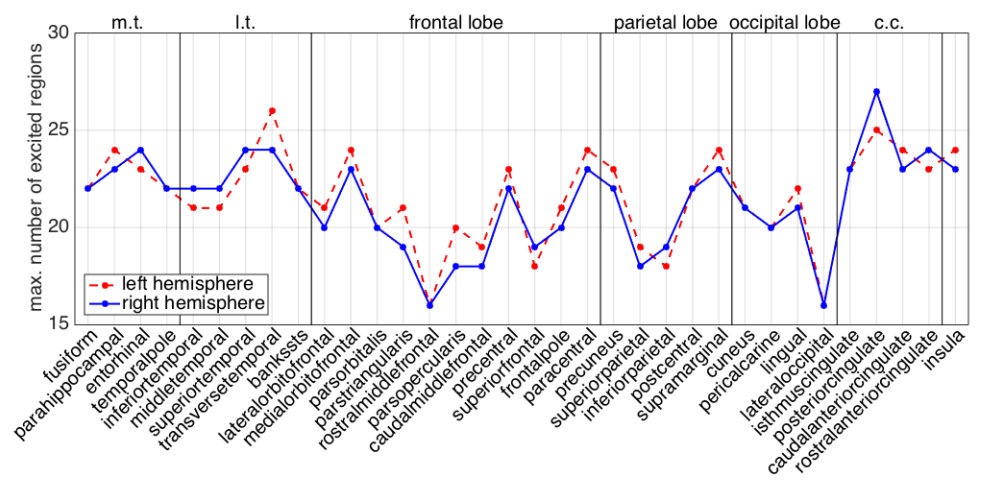

Fig. 5 Maximum number of excited regions. The maximum amount of excited regions for all the initially activated regions for the left (red dashed) and right (blue solid) hemisphere. The abbreviations give the affiliation to the lobes.

nected to the propagation of CSD on the real geometry of human brain. Our results may also be useful for the automatic identification of CSD from electrophysiological recordings [19].

Acknowledgements This work was supported by the Bizkaia Talent and European Commission through COFUND under the grant BRAhMS - Brain Aura Mathematical Simulation- (AYD-000285), and also by the Basque Government through the BERC 2014-2017 program, and by the Spanish Ministry of Economics and Competitiveness MINECO: BCAM Severo Ochoa excellence accreditation SEV-2013-0323. JMC acknowledges financial support from Ikerbasque: The Basque Foundation for Science and Euskampus at UPV/EHU.

\section{References}

1. Kroos, J.M., Diez, I., Cortes, J.M., Stramaglia, S., Gerardo-Giorda, L. (2016) Geometry Shapes Propagation: Assessing the Presence and Absence of Cortical Symmetries through a Computational Model of Cortical Spreading Depression. Front. Comput. Neurosci. 10:6. doi: 10.3389/fncom.2016.00006

2. Leão, A. (1944). Spreading depression of activity in the cerebral cortex. J. Neurophysiol. 7 , 391-396

3. Leão, A. (1947). Further observations on the spreading depression of activity in the cerebral cortex. J. Neurophysiol. 10, 409-414

4. de Tommaso, M., Ambrosini, A., Brighina, F., Coppola, G., Perrotta, A., Pierelli, F., Sandrini, G., Valeriani, M., Marinazzo, D., Stramaglia, S., Schoenen, J. (2014). Altered processing of sensory stimuli in patients with migraine. Nat Rev Neurol 10, 144-155

5. Hadjikhani, N., Sanchez del Rio, M., Wu, O., Schwartz, D., Bakker, D., Fischl, B., Kwong, K.K., Cutrer, F.M., Rosen, B.R., Tootell, R.B.H., Sorensen, A.G., Maskowitz, M.A. (2001). Mechnisms of migraine aura revealed by functional mri in human visual cor- 
tex. Proceedings of the National Academy of Sciences of the USA 98, 4687-4692. DOI: 10.1073/pnas.071582498

6. Richter, F., Lehmenkühler, A. (2008). Cortical spreading depression (csd): A neurophysiological correlate of migraine aura. Der Schmerz 22, 544-50. DOI: 10.1007/s00482-008-0653-9

7. M. Sramka, G. Brozek, J. Bures, P. Nadvornik (1977) Functional ablation by spreading depression: possible use in human stereotactic neurosurgery. Appl. Neurophysiol. 30 589-96

8. Costa, C., Tozzi, A., Rainero, I., Cupini, L.M., Calabresi, P., Ayata, C., Sarchielli, P. (2013) Cortical spreading depression as a target for anti-migraine agents, The Journal of Headache and Pain, 14:62, doi: 10.1186/1129-2377-14-62.

9. Dahlem, M., Schmidt, B., Bojak, I., Boie, F., Kneer, S., Hadjikhani, N., Kurths, J. (2015). Cortical hot spots and labyrinths: why cortical neuromodulation for episodic migraine with aura should be personalized. Front Comput Neurosci. 9. DOI: 10.3389/fncom.2015.00029

10. Cortes, J., Marinazzo, D., Series, P., Oram, M., Sejnowski, T., van Rossum, M. (2012). The effect of neural adaptation on population coding accuracy. Journal of Computational Neuroscience 32, 387-402. DOI: 10.1007/s10827-011-0358-4

11. Rogers, J., McCulloch, A. (1994). A collocation - galerkin finite element model of cardiac action potential propagation. IEEE Trans Biomed Eng. 41, 743-757. DOI: 10.1109/10.310090

12. FitzHugh, R. (1961). Impulses and physiological states in theoretical models of nerve membrane. Biophysical Journal 1, 445-466

13. Porooshani, H., Porooshani, A., Gannon, L., Kyle, G. (2004). Speed of progression of migrainous visual aura measured by sequential field assessment. Neuro-Ophthalmology 28, 101-105. DOI: 10.1076/noph.28.2.101.23739

14. Quarteroni, V., Valli, A. Numerical Approximation of Partial Differential Equations. Springer-Verlag, Berlin, 1994.

15. Fischl, B. (2012). Freesurfer. Neuroimage 62, 774-781

16. Diez, I., Bonifazi, P., Escudero, I., Mateos, B., Muñoz, M.A., Stramaglia, S., Cortes, J.M. (2015). A novel brain partition highlights the modular skeleton shared by structure and function. Sci Rep 5, 10532

17. Brodmann, K. (2006). Brodmann's Localisation in the Cerebral Cortex - The Principles of Comparative Localisation in the Cerebral Cortex Based on Cytoarchitectonics (Springer)

18. Saad, Y. (2003). Iterative methods for sparse linear systems. SIAM.

19. Yoshida, K., Xu, M., Natsubori, A., Mimura, M., Takata, N., Tanaka, K.F. (2015) Identification of the extent of cortical spreading depression propagation by Npas4 mRNA expression. Neurosci Res. 98:1-8. doi: 10.1016/j.neures.2015.04.003. 